\title{
Associations of age and sex with brain volumes and asymmetry in 2-5-week-old infants
}

\author{
S. J. Lehtola ${ }^{1}$ • J. J. Tuulari ${ }^{1} \cdot$ L. Karlsson ${ }^{1,2} \cdot$ R. Parkkola $^{3} \cdot$ H. Merisaari ${ }^{4} \cdot$ J. Saunavaara ${ }^{5} \cdot$ T. Lähdesmäki $^{6}$. \\ N. M. Scheinin ${ }^{1,7} \cdot$ H. Karlsson ${ }^{1,7}$
}

Received: 22 May 2018 / Accepted: 30 October 2018 / Published online: 2 November 2018

(c) The Author(s) 2018

\begin{abstract}
Information on normal brain structure and development facilitates the recognition of abnormal developmental trajectories and thus needs to be studied in more detail. We imaged 68 healthy infants aged 2-5 weeks with high-resolution structural MRI (magnetic resonance imaging) and investigated hemispheric asymmetry as well as the associations of various total and lobar brain volumes with infant age and sex. We found similar hemispheric asymmetry in both sexes, seen as larger volumes of the right temporal lobe, and of the left parietal and occipital lobes. The degree of asymmetry did not vary with age. Regardless of controlling for gestational age, gray and white matter had different age-related growth patterns. This is a reflection of gray matter growth being greater in the first years, while white matter growth extends into early adulthood. Sexdependent differences were seen in gray matter as larger regional absolute volumes in males and as larger regional relative volumes in females. Our results are in line with previous studies and expand our understanding of infant brain development.
\end{abstract}

Keywords Brain asymmetry $\cdot$ Infant brain $\cdot$ MRI $\cdot$ Brain volume $\cdot$ Infant age $\cdot$ Sexual dimorphism

\section{Introduction}

The development of the central nervous system (CNS) is a rapid and plastic process during the first years of life and is influenced by many intrinsic and extrinsic factors (Holland et al. 2014; Stiles and Jernigan 2010). During this sensitive

\section{S. J. Lehtola}

sajole@utu.fi

$1 \quad$ FinnBrain Birth Cohort Study, Turku Brain and Mind Center, Institute of Clinical Medicine, University of Turku, Lemminkäisenkatu 3, 2nd floor, 20520 Turku, Finland

2 Department of Child Psychiatry, University of Turku and Turku University Hospital, Turku, Finland

3 Department of Radiology, University of Turku and Turku University Hospital, Turku, Finland

4 Department of Future Technologies, University of Turku, Turku, Finland

5 Department of Medical Physics, Turku University Hospital, Turku, Finland

6 Department of Pediatric Neurology, University of Turku and Turku University Hospital, Turku, Finland

7 Department of Psychiatry, University of Turku, Turku University Hospital, Turku, Finland developmental time period, an unfavorable environment can result in disruption of CNS growth, which may lead to alterations in brain structure or function that can manifest as pathological conditions later in life. Though the field of research on infant brain development is advancing rapidly, the ramifications of early structural variation are still under active investigation. The challenges of infant brain imaging studies are not only restricted to data acquisition and analysis but also its interpretation (Arthurs et al. 2012).

Despite the challenges of studying infant brain development, we argue that there are four main reasons for studying the topic more deeply. First, highly contradictory reports exist on the maturation process of the cerebral cortex, including both cortical thickening and thinning as a sign of maturation (Walhovd et al. 2016). Second, although previous studies have observed that longer gestation associates with larger infant brain volumes and, not surprisingly, with infant overall growth (Aanes et al. 2015; Bora et al. 2014; Dean et al. 2018; Haukvik et al. 2014; Holland et al. 2014; Knickmeyer et al. 2017; Mills and Tamnes 2014; Munakata et al. 2013; Peterson et al. 2000; Thompson et al. 2007; van Soelen et al. 2010; Østgård et al. 2014), most such studies compared preterm and term-born infants. Therefore, less is known about whether gestational length differences amongst 
term-born children influence differences in brain volumes. Third, sex differences are as yet understudied. A growing body of literature recognizes that male brains generally have larger intracranial volume (ICV) as well as gray and white matter volumes during development than female brains (Gilmore et al. 2007; Knickmeyer et al. 2008a, b, 2017; Thompson et al. 2007; Uematsu et al. 2012; van Soelen et al. 2010). In addition, male brains have been shown to grow faster than female brains during the first 3 months of life (Holland et al. 2014). Overall though, sex differences in infant brain volumes seem to be subtle, similarly to those seen in adults (Guo et al. 2016). Fourth, extant studies indicate that left-right asymmetries exist already in the fetal brain (Rajagopalan et al. 2011) and distinctive growth patterns can be seen in different parts of the neonatal brain (Dean et al. 2018; Deoni et al. 2011; Gilmore et al. 2007; Tanaka et al. 2013). However, some of the evidence is conflicting and thus the true developmental direction and location of these asymmetries and their relationships with sex and age are still unknown. Thus, further refinement of knowledge in this area is beneficial.

Overall then, as only few studies have been published on the effects of age and sex on neonate brain size and asymmetry, it is important to confirm these previous findings with replication and especially provide new information on the relationships between asymmetry and infant sex as well as age, as this interaction is a novel aspect in the field. Moreover, information on other important factors possibly influencing brain development, such as infant growth markers, is largely missing. Some connections between infant characteristics (lower birth weight and height and head circumference) and smaller brain volumes have been reported, but the strongest effects seem to arise within infant groups of restricted growth, for instance, preterm very-low-birthweight or small for gestational age neonates (Aanes et al. 2015; Dean et al. 2018; Knickmeyer et al. 2017; Mills and Tamnes 2014; Østgård et al. 2014). The influences of these factors on the term-born brain still need to be defined.

In this study, we investigated the associations of age and sex with brain size as well as with brain asymmetry in 68 2-5-week-old infants. We hypothesized that age, calculated from either due date or birth date (both have been employed in previous literature), is associated with larger brain size and that males have larger brain volumes than females. As a novel approach, we describe the asymmetry patterns and interregional correlations among brain volumes and lobar asymmetry patterns in relation to age and sex. We hypothesized that brain asymmetry is similar in both sexes and is seen as rightward (i.e., the right side lobe being larger) in frontal and temporal lobes and leftward (i.e., the left side lobe being larger) in parietal and occipital lobes, and also that asymmetry is more prominent in older infants. Additionally, we explored if closely located brain regions have the greatest interlobar volume correlations and if infant growth markers associate with brain volumes.

\section{Methods}

The study was conducted according to the Declaration of Helsinki, and was reviewed and approved by the Ethics Committee of the Hospital District of Southwest Finland.

\section{Participants}

Participants were 68 healthy Finnish (Caucasian) neonates (31 males, 37 females) from 2 to 5 weeks of age (counted from the estimated due date). All the infants were born fullterm [between gestational weeks (gwks) 37 and 42] and weighed more than $2500 \mathrm{~g}$. The exclusion criteria for the infants were: occurrence of any perinatal complications with neurological consequences (e.g., hypoxia), scoring lower than 5 points in the 5 min Apgar, previously diagnosed CNS anomaly or an abnormal finding in a previous MRI scan. The families were contacted via telephone and eligibility for the study was assessed. After explaining the purpose and protocol of the study, a written informed consent was signed by the parents on behalf of each infant.

\section{Clinical characteristics}

The descriptive information of the infant population is presented in Table 1. No sex differences were found regarding the duration of pregnancy; infant age at scan counted from the estimated due date or from the birth date; birth weight or birth length; head circumference; duration of the labor; maternal pre-pregnancy body-mass-index (BMI) or age at term, or paternal age at term; maternal smoking or antidepressive medication in the first or last trimester; or maternal education.

\section{Obstetric data and demographic information}

Obstetric data were retrieved from the Finnish Medical Birth Register of the National Institute for Health and Welfare (http://www.thl.fi), and included length of pregnancy, gestational age at birth, head circumference, birthweight and birth length. Additional information was also collected as a part of the FinnBrain Study protocol, such as maternal pre-pregnancy body-mass-index (BMI) and maternal and paternal age.

\section{MRI acquisition}

The participants underwent the MRI scans solely for research purposes and without clinical indications. The 
Table 1 Data descriptives

\begin{tabular}{|c|c|c|c|c|c|}
\hline Variables & $\begin{array}{l}\text { All subjects }(n=68) \text {, } \\
\text { mean }(\mathrm{SD})\end{array}$ & $\begin{array}{l}\text { Boys }(n=31), \\
\text { mean }(\mathrm{SD})\end{array}$ & $\begin{array}{l}\text { Girls }(n=37), \\
\text { mean }(\mathrm{SD})\end{array}$ & $F$ & $p$ \\
\hline Infant age at scan (from estimated due date) (d) & $25.6(8.1)$ & $23.9(7.6)$ & $27.0(8.3)$ & 0.826 & 0.12 \\
\hline Infant age at scan (from birth date) (d) & $25.5(7.2)$ & $25.3(7.8)$ & $25.2(6.8)$ & 2.33 & 0.928 \\
\hline Birthweight $(\mathrm{g})$ & $3564(429)$ & $3603(403)$ & $3531(454)$ & 0.526 & 0.494 \\
\hline Birth length (cm) & $50.3(1.7)$ & $50.6(1.7)$ & $50.1(1.7)$ & 0.448 & 0.287 \\
\hline Head circumference $(\mathrm{cm})$ & $35.2(1.3)$ & $35.5(1.2)$ & $35(1.3)$ & 0.555 & 0.169 \\
\hline Duration of labor (min) & $514(293)$ & $506(291)$ & $523(299)$ & 0.014 & 0.819 \\
\hline Maternal prepregnancy BMI $\left(\mathrm{kg} / \mathrm{m}^{2}\right)$ & $24.3(3.9)$ & $23.4(3.4)$ & $25(4.1)$ & 1.267 & 0.095 \\
\hline Maternal age at term $(\mathrm{y})$ & $30.2(4.5)$ & $30.9(4.3)$ & $29.6(4.6)$ & 0.005 & 0.251 \\
\hline Paternal age at term (y) & $31.6(4.4)$ & $30.8(3.6)$ & $32.2(4.8)$ & 1.423 & 0.279 \\
\hline Maternal education $^{\mathrm{a}}$ & Number $(\%)$ & Number $(\%)$ & Number $(\%)$ & $1.454^{\mathrm{b}}$ & 0.483 \\
\hline (1) Elementary school & $1(1.5)$ & $1(3.2)$ & $0(0)$ & & \\
\hline (2) High school/career college & $41(62.1)$ & $20(64.5)$ & $21(60)$ & & \\
\hline (3) College/university & $24(36.4)$ & $10(32.3)$ & $14(40)$ & & \\
\hline Maternal smoking 1st trimester & $7(10.3)$ & $3(9.7)$ & $4(10.8)$ & $0.023^{\mathrm{b}}$ & 0.878 \\
\hline Maternal smoking 3rd trimester & $3(4.4)$ & $1(3.3)$ & $2(6.1)$ & $0.258^{\mathrm{b}}$ & 0.612 \\
\hline Maternal medication 1st trimester & $5(7.4)$ & $4(12.9)$ & $1(2.7)$ & $2.576^{\mathrm{b}}$ & 0.108 \\
\hline Maternal medication 3rd trimester & $3(4.4)$ & $3(10.3)$ & $0(0)$ & $3.693^{\mathrm{b}}$ & 0.055 \\
\hline
\end{tabular}

Results of independent samples $t$ tests: no significant differences between the sexes within the variables listed below

${ }^{a}$ Maternal medication $=$ medication against depression/anxiety during pregnancy

${ }^{\mathrm{b}}$ Note small subsample sizes

participants were imaged with a Siemens Magnetom Verio 3T scanner (Siemens Medical Solutions, Erlangen, Germany). The imaging protocol was $40 \mathrm{~min}$ long and included an axial PD-T2-TSE (Dual-Echo Turbo Spin Echo) sequence, a sagittal 3D-T1 (T1-weighted MPRAGE) sequence, and diffusion tensor imaging (DTI) sequence. Only T2-weighted images were used in the current study, as they provide a good contrast between the skull and the brain. The slice thickness of the PD-T2 sequence was only $1 \mathrm{~mm}$, to achieve an isotropic voxel size of $1.0 \mathrm{~mm}^{3}$. TR (time repetition) time of $12,070 \mathrm{~ms}$ and effective TE (time echo) times of $13 \mathrm{~ms}$ and $102 \mathrm{~ms}$ were used to produce both PD-weighted and T2-weighted images from the same acquisition. The total number of slices was 128. Sequence parameters were optimized so that "whisper" gradient mode could be used in PD-T2 TSE and 3D-T1 sequences to reduce acoustic noise during the scan.

The scanning was performed at the Medical Imaging Centre of the Hospital District of Southwest Finland mostly during afternoon and evening hours though some scans occurred during the day on weekends. An experienced radiographer received the family, reviewed the imaging protocol with them, and confirmed the absence of any safety risks (inner ear implants, pacemakers or other metal devices or parts). The imaging was performed without anesthesia, during natural sleep, before which the infants were fed with milk (breast milk or formula) and swaddled into sleep in a vacuum mattress. The parents were able to be present in the scanning room during the whole scan and had the opportunity to quit the study at any point of the procedure. Both the parents and the infant were provided with sufficient hearing protection (ear plugs or wax and custom-sized ear muffs for all infants). The radiographer observed the scanning through a window with a microphone contact to a parent and a loudspeaker with which all the sounds from the scanning room were heard, which allowed the radiographer to know if the infant woke. A scanning session was ended if the baby did not fall asleep before or during the scan. After the scan, a small thank you gift was given to the family (a bop hat or body suit) for participation.

All the brain images were assessed by a pediatric neuroradiologist for any incidental findings and, if found to have one, the infant and the parents were given a chance for a follow-up by a pediatric neurologist. Developmental status has thereafter been normal for all of the participants, including those with incidental findings (the manuscript describing the incidental findings in greater detail has been submitted to a peer-reviewed journal), who were included in the study. The incidental findings were deemed not to affect brain volume estimates and have been found to be common and clinically insignificant in previous studies (Rooks et al. 2008; Whitby et al. 2004). 


\section{Image preprocessing}

The data were analyzed in iBEAT (Dai et al. 2013), an open source toolbox for processing infant brain images. After file conversion from dicom to analyze format, the images were preprocessed, which included reorientation and subject to N3 intensity non-uniformity correction. Then the skull was extracted with an iBEAT module and, if the extraction was incomplete, manual correction of the brain mask was done. Next, tissue segmentation, i.e., separation of gray and white matter [and cerebrospinal fluid (CSF)], was performed (Fig. 1). Finally, iBEAT labeled the different parts of the brain according to an infant-specific Automatic Anatomical Labeling (AAL) atlas. Total gray matter volume (TGM) was counted using the iBEAT segmented and AAL labeled data. While the cortical segmentation was performed successfully, the subcortical segmentation output contained parts of the white matter and was thus excluded from the analyses.
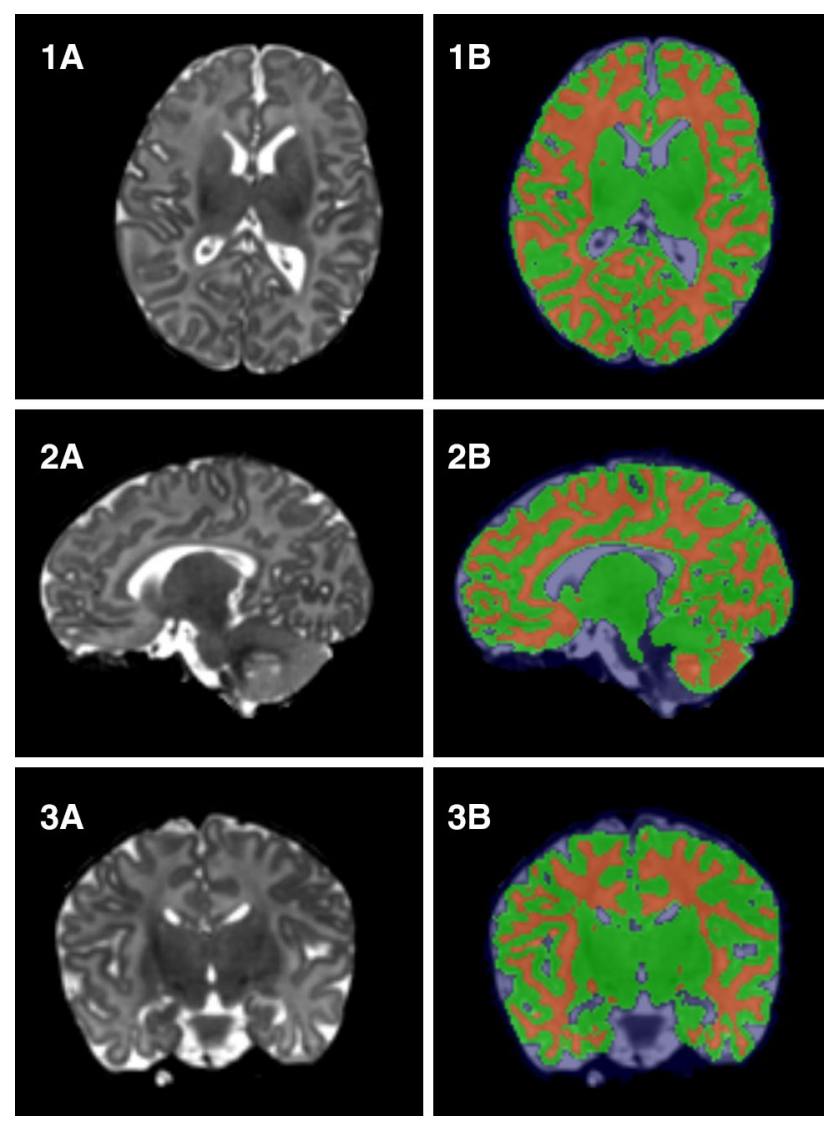

Fig. 1 Representatives of (1) sagittal, (2) axial and (3) coronal views generated from a subject's T2-weighted image. 1A-3A are the original $\mathrm{T} 2$-weighted images and $1 \mathrm{~B}-3 \mathrm{~B}$ are the segmented images, where the gray matter is marked with green and the white matter by red

\section{Brain volume calculation}

Total brain volume (TBV) was calculated as a combination of all gray and white matter volumes, and intracranial volume (ICV) was calculated by adding cerebrospinal fluid (CSF) to TBV. Total cortical volume (TCV) was calculated by subtracting the subcortical parts from TGM. Lobar volumes were calculated using the AAL labeled data by combining together the volumes of separate anatomical brain parts belonging to a particular lobe. Ratios of the gray and white matter and lobar volumes in relation to total brain volumes, namely relative volumes, were also calculated for total white matter (TWM), TGM, TCV and lobar volumes so that TGM, TWM and TCV were expressed as relative to TBV and lobar volumes to TGM as they contained only gray matter.

\section{Statistical analyses}

The IBM SPSS Statistics Version 23 for MAC was used for statistical analyses (Armonk, NY: IBM Corp.). The normality of the data was checked by visual confirmation and with Shapiro-Wilk test, and $p$ values smaller than 0.05 were considered statistically significant, unless otherwise noted. To prevent possible Type I errors due to multiple tests, a Bonferroni correction was applied for all the analyses including multiple comparisons.

\section{Associations with age and infant demographics}

Infant age at scan varied if counted from the estimated due date or from the actual birth date, and thus, both ages were calculated and the associations between infant age and brain volumes were analyzed separately for both age variables using bivariate correlations. These results were then controlled for sex with partial correlation. The relationships between infant growth markers (head circumference, birth weight and length) and brain volumes were investigated with bivariate and partial correlations (controlling for sex and age).

\section{Sex differences}

To analyze differences in clinical variables between sex groups, a parametric test ( $t$ test) was used as the data was not found to deviate from normal distribution. Sex differences in mean brain volumes were examined with the independent samples $t$ test. Simple linear regression, including age-bysex interaction, was run to examine associations between gestational corrected age and brain volumes having sex as a fixed factor. All the analyses were repeated after excluding the infants $(N=5)$ exposed to maternal antidepressive medication during pregnancy and this did not affect the results. 


\section{Asymmetry}

An asymmetry index (AI) (Dean et al. 2018; Tanaka et al. 2013; Uematsu et al. 2012) was calculated for each individual with the formula [(left - right $/($ left + right $)) \times 100]$. Positive values represented leftward (left $>$ right) and negative values rightward asymmetry. The statistical significance of hemispheric asymmetry was calculated using a one sample $t$ test (testing against the reference value 0.00). If asymmetry was detected, differences between sexes were tested with independent samples $t$ test. Simple linear regression was used to examine the relationship between asymmetry and age with sex as a covariate. Finally, bivariate correlations were implemented to detect possible correlations within regional brain volumes.

\section{Results}

\section{Mean brain volumes}

The mean intracranial volume (ICV) for all subjects was 616 (SD 57.9) $\mathrm{ml}$, the mean total brain volume (TBV) was 446 (SD 41.7) $\mathrm{ml}$, the mean total gray matter volume (TGM) was 238 (SD 24.8) $\mathrm{ml}$, the mean total white matter volume (TWM) was 208 (SD 18.4) $\mathrm{ml}$ and the mean total cortical volume (TCV) was 221 (SD 23.6) $\mathrm{ml}$. The TGM volumes were highly similar in each hemisphere $\left(119 \mathrm{~cm}^{3} \mathrm{SD}\right.$ 12.4) (Table 2; Fig. 2). The mean volume of the right side of the temporal lobe was greater than that of the left side ( $p<0.001$, Bonferroni corrected for multiple comparisons) and the volume of the left side of the occipital lobe was greater than that of the right side $(p<0.001$, Bonferroni corrected for multiple comparisons) (Fig. 2).

\section{Interlobar correlations}

Correlation analysis revealed that ICV correlated strongly with all the major tissue compartment volumes (TBV, TWM, TCV), which were also strongly correlated with each other (Fig. 3). Strong correlations between TGM and the following structures were also seen: right and left TGM; total, left and right volumes of frontal, parietal, temporal and occipital lobes. All lobar volumes were strongly correlated with each other. Interestingly, the strongest correlations in the analysis were between the temporal regions and the other lobes. TWM showed poorer correlation with frontal and parietal lobes and TCV with parietal lobe (Fig. 3).

\section{Correlations between infant growth metrics and brain volumes}

With absolute volumes, head circumference was expectedly positively correlated with ICV, TBV, TWM and TCV (Table 3). Comparison with relative brain volumes showed a
Table 2 Mean brain volumes and the percentages relative to total volume

\begin{tabular}{lcc}
\hline Cerebral structures & $\begin{array}{l}\text { Mean volume in ml, } \\
\text { (SD) }\end{array}$ & $\begin{array}{l}\text { \% of total } \\
\text { volume }^{\mathrm{a}}\end{array}$ \\
\hline TWM & $208(18.4)$ & 46.6 \\
TGM & $238(24.8)$ & 53.4 \\
Left & $119(12.4)$ & 26.7 \\
Right & $119(12.4)$ & 26.7 \\
TCV & $221(23.6)$ & 49.6 \\
Frontal lobe & $78.3(9.1)$ & 17.5 \\
Left & $39(4.6)$ & 8.7 \\
Right & $39.2(4.6)$ & 8.7 \\
Parietal lobe & $42.3(4.7)$ & 9.5 \\
Left & $21.3(2.4)$ & 4.8 \\
Right & $20.9(2.4)$ & 4.7 \\
Temporal lobe & $46.5(5.0)$ & 10.4 \\
Left & $21.4(2.3)$ & 4.8 \\
Right & $25.2(2.8)$ & 5.7 \\
Occipital lobe & $53.7(6.3)$ & 12 \\
Left & $33.8(4.0)$ & 7.6 \\
Right & $25(2.9)$ & 5.6 \\
\hline
\end{tabular}

$I C V$ intracranial volume, $T B V$ total brain volume, $T W M$ total white matter, $T G M$ total gray matter volume, $T C V$ total cortical volume

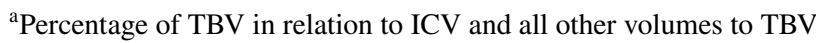

positive relationship between head circumference and frontal lobe volume and a negative relationship between head circumference and occipital lobe volume. No correlations were found between absolute or relative brain volumes and birth weight or length. After controlling for sex and age (counted from due date), all these findings persisted, and additionally head circumference was positively associated with absolute frontal lobe volume and negatively with total occipital lobe volume. Birth length was positively associated with absolute TGM (Table 3).

\section{Effects of neonate age on brain volumes}

No significant correlations were observed between the two calculated ages at scan and absolute total or lobar brain volumes in the study group as a whole (Table 4), but after controlling for sex, moderate positive correlations were seen between age counted from the estimated due date and TCV as well as total and right parietal lobe volumes. The correlations, separately for the subgroups of males and females, are presented in Table 4. The same analyses with relative brain volumes in the whole group showed a positive correlation between age and TGM as well as TCV and a negative correlation with TWM, regardless of if counting from the estimated due date or the actual birth date (Fig. 4). Table 4 provides a detailed report on the correlations. 

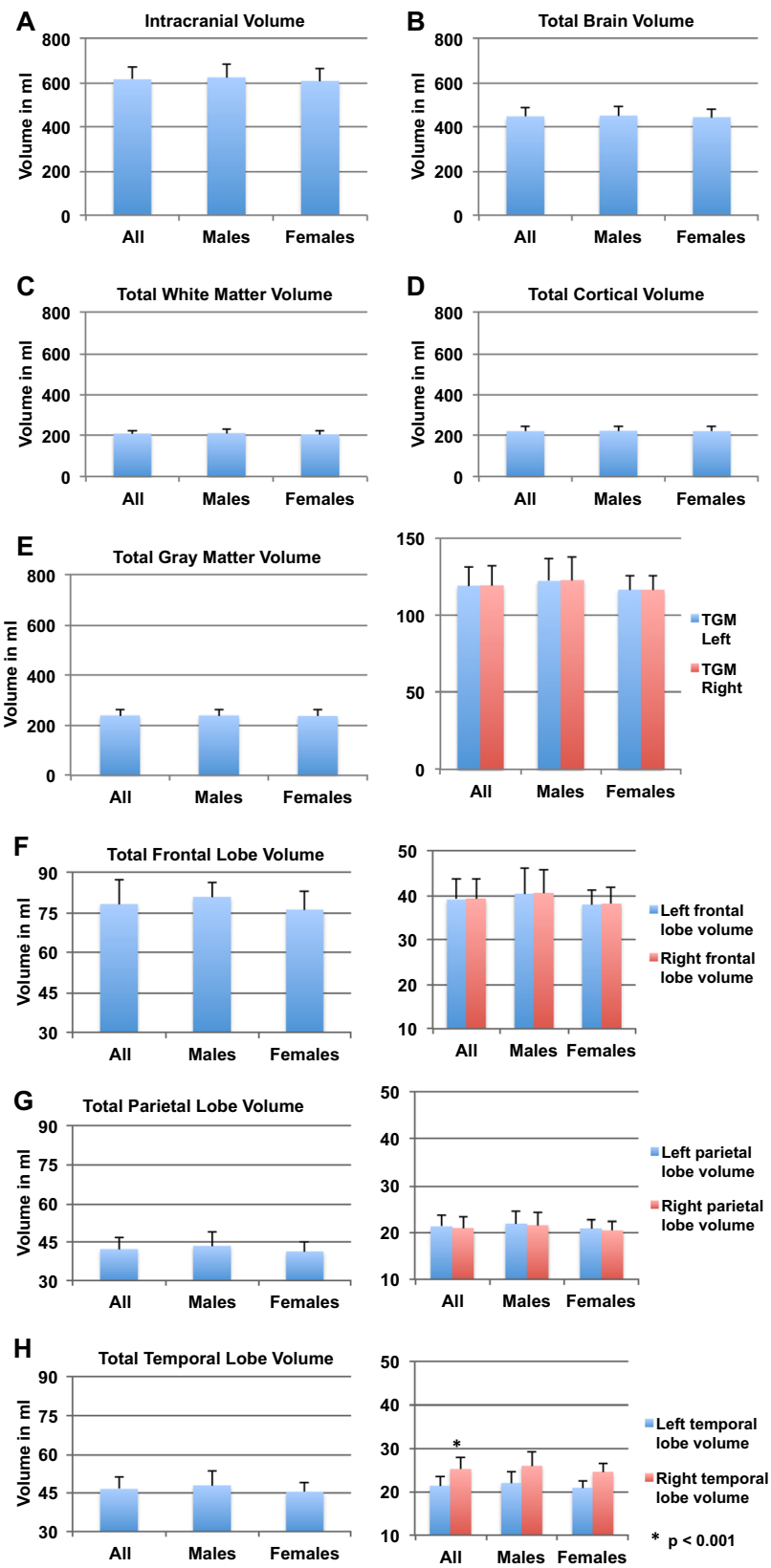

I Total Occipital Lobe Volume
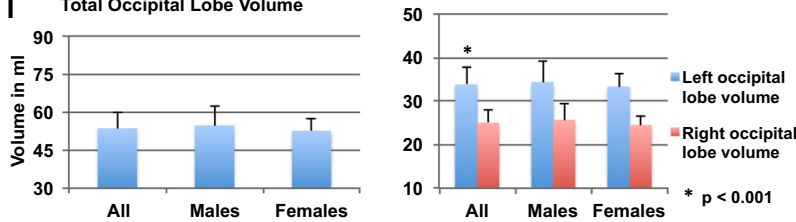

Fig. 2 Mean total brain volumes ( $\mathrm{ml})$ in all subjects, males and females. a Mean intracranial volume. b Mean total brain volume. c Mean total white matter volume. d Mean total cortical volume. In the following, the left side represents the total volumes and the right side the hemispheric volumes in all subjects, males and females: e mean total gray matter volume, $\mathbf{f}$ frontal lobe volumes, $\mathbf{g}$ parietal lobe volumes, $\mathbf{h}$ temporal lobe volumes, i occipital lobe volumes. Statistically significant ( $p<0.001$, Bonferroni corrected for multiple comparisons) findings are marked by an asterisk and standard deviations by bars

\section{Sex differences in brain volumes}

A significant difference was found between the sexes in the absolute total volumes of the frontal and temporal lobes, so that in these structures, males had greater volumes (Table 5). A similar difference was seen in the right hemisphere and also in TGM. For the left hemisphere, this effect was only seen in the temporal lobe (Table 5). After correcting TGM with TVB and the lobar volumes with TGM, the results revealed a significant sex difference in TGM, the total volume of the parietal lobe, the volume of the right temporal lobe and the left parietal lobe so that females had greater relative volumes in these structures than males. No results survived the correction for multiple comparisons (Bonferroni, $p<0.006$ ) (Table 5).

\section{Asymmetry}

The absolute volume of the left parietal lobe was $0.9 \%$ larger than the right in the whole group $(p=0.003)$. The right temporal and the left occipital lobes were significantly larger (8\% and 16\%) than the other side in the whole group $(p<0.0001)$ (Fig. 5). Asymmetry patterns were found to be similar in both sexes. All these findings persisted when corrected with TGM volume (Table 6). Regression analysis with sex as a fixed factor, and age as an independent factor revealed that age was not associated with the degree of asymmetry.

\section{Discussion}

\section{Interlobar correlations}

Our correlation analysis revealed that although all lobar volumes were highly correlated with each other, the temporal regions had the highest correlation with all other lobar regions while the occipital region had the weakest relationships with the right frontal and the left parietal lobes. While the anatomical structure of the brain can change as a result of functional specialization (Lerch et al. 2006), one could expect that the way the anatomical regions are correlated might reflect the functional interaction between these brain structures, and that a change in the correlations might serve as a developmental marker in addition to asymmetry. The strong connection of other brain regions to the temporal lobes is logical as they execute many important sensory information processing and cognitive functions. However, we acknowledge that correlations are a statistical construct that reflect connectivity information to a very limited extent (Irimia and Van Horn 2013) and thus by reporting them did not aim to describe connectomics. It is clear that to draw further conclusions on the interrelationships between 
Fig. 3 Intercorrelations of brain volumes. Colors indicate the value of the correlation coefficient and the strength of the relationship between brain volumes. Asterisks designate the statistical significance level $(* p<0.05 ; * * p<0.001)$

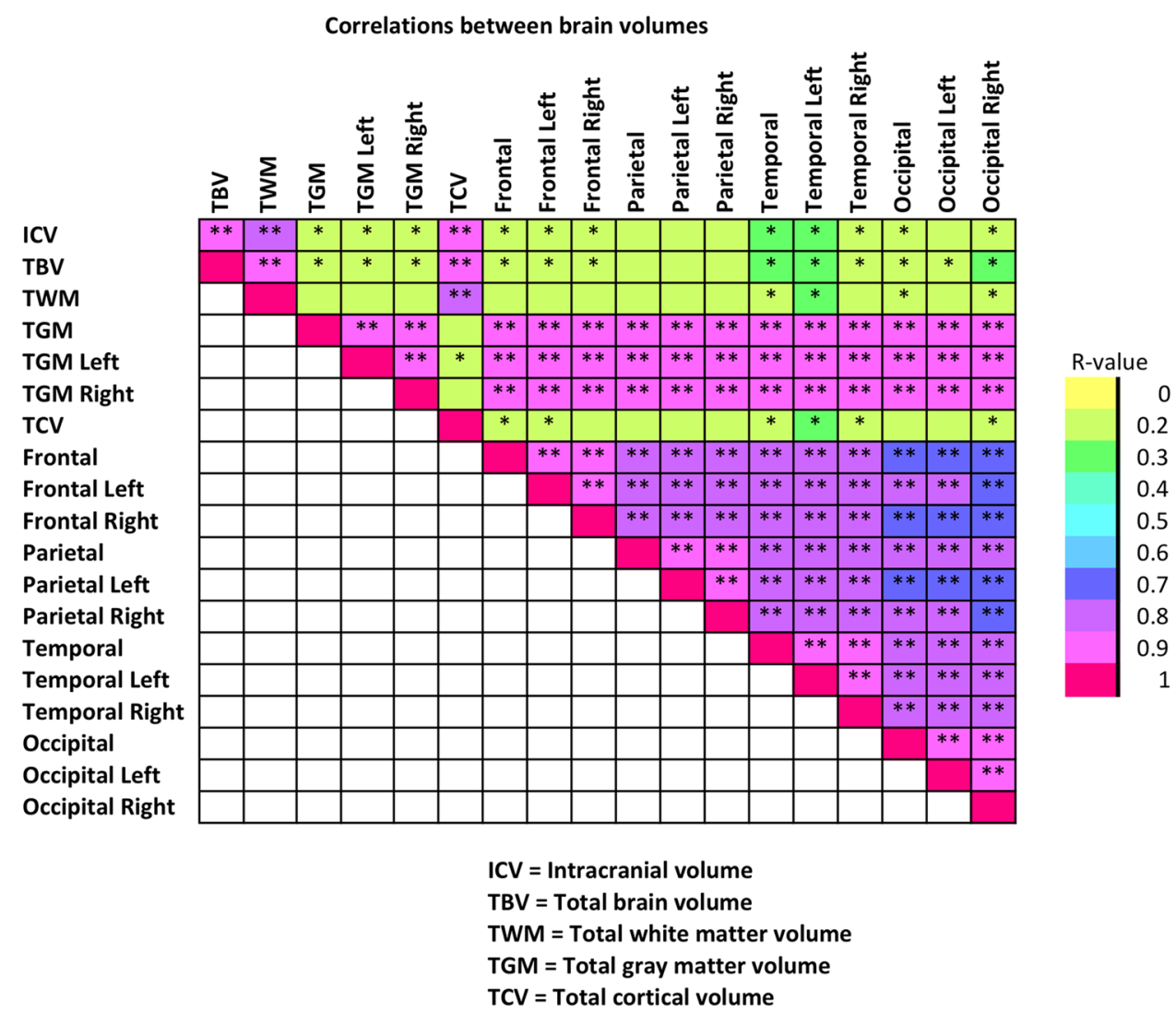

structures and function would require the use of additional data sources.

\section{Brain volume relations with infant body size}

Concerning neonatal growth markers, including birth weight, birth length and head circumference, both sex groups had similar results (Table 4). Of these infant metrics, greater head circumference was expectedly associated with larger absolute volumes of ICV, TBV, TWM, TCV, but also with larger absolute and relative volumes of frontal lobes, and smaller relative volumes of the occipital lobe. We found no correlation between birth weight or length and brain volumes, but after controlling for sex, a positive correlation was seen between birth length and TWM. The effect of body size should always be evaluated and, if necessary, acknowledged in the analyses, especially in special prenatal conditions that could influence growth (Walhovd et al. 2012), although our sample consisted of only healthy, term-born individuals.

\section{Brain volume relations with age}

Age expectedly associated positively with the size of TGM and TCV and negatively with that of TWM in both sexes. In addition, in males, age predicted positively also the absolute volumes of ICV and TBV. As for the negative association between age and TWM in females, it has been reported before (Knickmeyer et al. 2008a, b), where the effect was seen during a follow-up of 24 months; yet, the majority of the evidence regarding the effect of age on both gray and white matter volume indicates the relationships to be positive (Bora et al. 2014; Holland et al. 2014; Peterson et al. 2000). Gray matter volume increases during the first years of life, while previous studies have argued that the growth of white matter is steadier, but likely extends into adulthood (Knickmeyer et al. 2008a, b). Our results support this by showing that white matter growth is slower compared to that of gray matter in the early stages of life.

In contrast to the previous findings, a recent large study ( $n=756)$ (Knickmeyer et al. 2017) on the effects of demographic and obstetric factors on infant brain volumes, detected that infants born earlier had larger brain volumes than later born infants (gestational age at birth ranging from 27 to 46 weeks). Importantly though, this finding was only apparent when the volumes were adjusted to gestational age at MRI and birth weight. The authors speculated this to be the result of accelerated brain growth happening in the premature brain after birth. Nevertheless, infants born earlier had some smaller volumes in the visual association cortex, primary auditory, and auditory association cortex, which is in line with previous studies on premature infants (Knickmeyer et al. 2017). This latter finding of shorter gestation 
Table 3 Comparison of absolute and relative brain volumes and infant growth markers

\begin{tabular}{|c|c|c|c|c|c|c|c|c|c|c|c|c|}
\hline \multirow[t]{3}{*}{ Brain structures } & \multicolumn{4}{|c|}{ Head circumference } & \multicolumn{4}{|c|}{ Birth weight } & \multicolumn{4}{|c|}{ Birth length } \\
\hline & \multicolumn{2}{|c|}{ Absolute } & \multicolumn{2}{|l|}{ Relative } & \multicolumn{2}{|c|}{$\begin{array}{l}\text { Abso- } \\
\text { lute }\end{array}$} & \multicolumn{2}{|c|}{ Relative } & \multicolumn{2}{|c|}{ Absolute } & \multicolumn{2}{|c|}{ Relative } \\
\hline & $R$ & $p$ & $R$ & $p$ & $R$ & $p$ & $R$ & $p$ & $R$ & $p$ & $R$ & $p$ \\
\hline ICV & 0.319 & $0.008^{*}$ & & & - & - & & & - & - & & \\
\hline TBV & 0.319 & $0.009^{*}$ & & & - & - & & & - & - & & \\
\hline TWM & 0.340 & $\mathbf{0 . 0 0 5}^{*}$ & - & - & - & - & - & - & 0.269 & $0.029^{*}$ & - & - \\
\hline TGM & - & - & - & - & - & - & - & - & - & - & - & - \\
\hline Left & - & - & - & - & - & - & - & - & - & - & - & - \\
\hline Right & - & - & - & - & - & - & - & - & - & - & - & - \\
\hline TCV & 0.289 & $0.019^{*}$ & - & - & - & - & - & - & - & - & - & - \\
\hline Frontal & 0.262 & $0.039 *$ & 0.316 & $0.010^{*}$ & - & - & - & - & - & - & - & - \\
\hline Left & 0.254 & $0.033^{*}$ & 0.289 & $0.018^{*}$ & - & - & - & - & - & - & - & - \\
\hline Right & - & - & 0.312 & $0.011^{*}$ & - & - & - & - & - & - & - & - \\
\hline Parietal & - & - & - & - & - & - & - & - & - & - & - & - \\
\hline Left & - & - & - & - & - & - & - & - & - & - & - & - \\
\hline Right & - & - & - & - & - & - & - & - & - & - & - & - \\
\hline Temporal & - & - & - & - & - & - & - & - & - & - & - & - \\
\hline Left & - & - & - & - & - & - & - & - & - & - & - & - \\
\hline Right & - & - & - & - & - & - & - & - & - & - & - & - \\
\hline Occipital & - & - & -0.330 & $0.007^{*}$ & - & - & - & - & - & - & - & - \\
\hline Left & - & - & -0.272 & $0.027 *$ & - & - & - & - & - & - & - & - \\
\hline Right & - & - & -0.329 & $0.007^{*}$ & - & - & - & - & - & - & - & - \\
\hline
\end{tabular}

Pearson used for normally distributed values and Spearman for non-normally distributed values. Only statistically significant $(p<0.05)$ results are listed and marked with an asterisk. Bonferroni correction for multiple comparisons corresponding to $p<0.0058$. $p$ values meeting the significance criteria are bolded

$I C V$ intracranial volume, $T B V$ total brain volume, $T W M$ total white matter, $T G M$ total gray matter volume, $T C V$ total cortical volume, $R$ correlation coefficient, - no association/ non-significant being associated with smaller brain volumes, together with our results, in which greater age predicted larger brain volumes during the first weeks of postnatal life of term-born infants, support the earlier reports of the positive connection between brain volumes and longer gestation.

\section{Brain volume relations with sex}

Partly in conflict with previous evidence (Dean et al. 2018; Gilmore et al. 2007; Knickmeyer et al. 2017), we found no sex differences in ICV, TBV, TWM or TCV. However, neither did Gilmore when correcting for ICV (Gilmore et al. 2007). In our findings, TGM of the right hemisphere was greater in boys, but after controlling with TBV, the finding did not persist, thus supporting the findings of Munakata et al. on late-preterm and term infants showing no relationship between gray matter volume and sex (Munakata et al. 2013). Hence, the sex-related dimorphic volumetric differences might not be seen in the total volumes, but rather more regionally, balancing each other out so that the total volumes stay relatively equal. The diversion in results might also be the product of diverse sample sizes (Dean et al. 2018; Gilmore et al. 2007; Munakata et al. 2013).

As in previous research (Knickmeyer et al. 2008a, b; van Soelen et al. 2010), our results support the notion that male brains have greater absolute lobar volumes than females, specifically, the total and right side volumes of the frontal, temporal and parietal regions. Interestingly, females presented relatively larger volumes of TGM, of the total and left parietal lobes and of the right temporal lobe. These results align with the result of a previous study, done on children and young adults, showing greater gray matter to white matter ratio in females (Koolschijn and Crone 2013). However, the sex differences in our findings were modest and might not be of biological significance. Nevertheless, combining existing findings with our findings on the larger particular absolute volumes in males as well as the larger specific relative volumes in females provides support for theorizing that possible sex differences in the infant brain are more intricate and regional. However, the information concerning regional sexual dimorphism is still scarce and further definitive conclusions cannot be made. 
Table 4 Correlations between absolute and relative brain volumes and infant age at scan counted from estimated due date

\begin{tabular}{|c|c|c|c|c|c|c|}
\hline \multirow[t]{3}{*}{ Cerebral structures } & \multicolumn{3}{|c|}{ Absolute volumes } & \multicolumn{3}{|l|}{ Relative volumes } \\
\hline & All $(N=68)$ & Males $(N=31)$ & Females $(N=37)$ & All $(N=68)$ & Males $(N=31)$ & Females $(N=37)$ \\
\hline & $p(\mathrm{R})$ & $p(\mathrm{R})$ & $p(\mathrm{R})$ & $p(\mathrm{R})$ & $p(\mathrm{R})$ & $p(\mathrm{R})$ \\
\hline ICV & - & $\mathbf{0 . 0 0 2} *(0.540)$ & - & & & \\
\hline TBV & - & 0.001* $(0.552)$ & - & & & \\
\hline TWM & - & - & - & - & $<\mathbf{0 . 0 0 1} *(-0.637)$ & $0.005^{*}(-0.454)$ \\
\hline TGM & - & - & - & $<\mathbf{0 . 0 0 1} *(0.605)$ & $<\mathbf{0 . 0 0 1}^{*}(0.650)$ & 0.001* $(0.538)$ \\
\hline Left & - & - & - & $<\mathbf{0 . 0 0 1} *(0.576)$ & $<\mathbf{0 . 0 0 1} *(0.633)$ & 0.001* $(0.516)$ \\
\hline Right & - & - & - & $<\mathbf{0 . 0 0 1} *(0.480)$ & 0.001* $(0.578)$ & - \\
\hline $\mathrm{TCV}$ & - & $<\mathbf{0 . 0 0 1}^{*}(0.645)$ & - & $<\mathbf{0 . 0 0 1} *(0.559)$ & $<\mathbf{0 . 0 0 1}^{*}(0.682)$ & $0.006 *(0.446)$ \\
\hline Frontal lobe & - & - & - & - & - & $\mathbf{0 . 0 0 2} *(-0.486)$ \\
\hline Left & - & - & - & - & - & $0.004 *(-0.465)$ \\
\hline Right & - & - & - & - & - & $0.004 *(-0.461)$ \\
\hline Parietal lobe & - & - & - & - & - & - \\
\hline Left & - & - & - & - & - & - \\
\hline Right & - & - & - & - & - & - \\
\hline Temporal lobe & - & - & - & - & - & - \\
\hline Left & - & - & - & - & - & - \\
\hline Right & - & - & - & - & - & - \\
\hline Occipital lobe & - & - & - & - & - & $0.030 *(0.357)$ \\
\hline Left & - & - & - & - & - & $0.017 *(0.390)$ \\
\hline Right & - & - & - & - & - & - \\
\hline
\end{tabular}

Pearson has been used for normally distributed values and Spearman for non-normally distributed values. Only statistically significant $(p<0.05)$ results are listed and marked with an asterisk. Bonferroni correction for multiple comparisons corresponding to $p<0.0028$. $p$ values meeting the significance criteria are bolded

$D D$ age counted from estimated due date, $I C V$ intracranial volume, $T B V$ total brain volume, $T G M$ total gray matter volume, $T C V$ total cortical volume, - no correlation/non-significant, $I C V$ intracranial volume, $T B V$ total brain volume, $T W M$ total white matter, $T G M$ total gray matter volume, $T C V$ total cortical volume
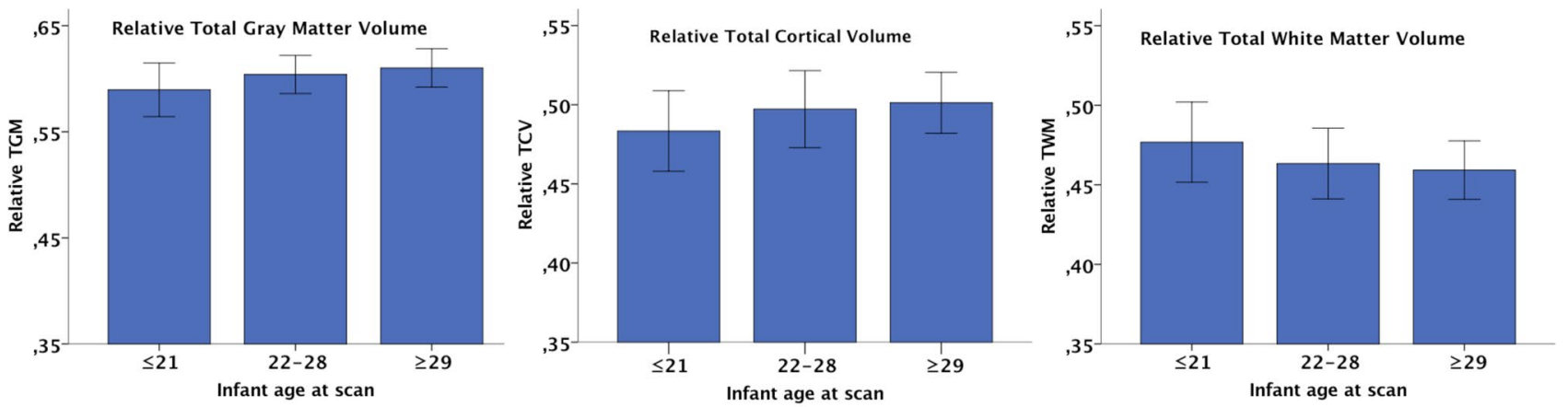

Fig. 4 Relative total volumes and age at scan in days counted from estimated due date $(p<0.001)$. a Relative total gray matter volume; $\mathbf{b}$ relative total cortical volume; $\mathbf{c}$ relative total white matter volume. The bars represent standard deviations

\section{Brain asymmetry}

We detected similar lobar asymmetry in both sexes: rightward in the temporal lobe and leftward in parietal and occipital lobes. This right-larger-than-left asymmetry in temporal lobe is strongly consistent with previous research on early asymmetry (Dean et al. 2018; Mark et al. 1999; Matsuzawa et al. 2001; Rajagopalan et al. 2011; Tanaka et al. 2013) and although it has been hypothesized to be associated with the development of speech and language, its presence in the "prespeech population" of newborns defends the theory that brain asymmetry exists already in the infant brain before the 
Table 5 Sex differences in mean absolute and relative brain volumes

\begin{tabular}{|c|c|c|c|c|c|c|c|c|}
\hline \multirow[t]{2}{*}{ Cerebral structures } & \multicolumn{4}{|c|}{ Mean absolute volumes } & \multicolumn{4}{|c|}{ Mean relative volumes } \\
\hline & $\begin{array}{l}\text { Males }(n=31) \\
\mathrm{cm}^{3}(\mathrm{SD})\end{array}$ & $\begin{array}{l}\text { Females } \\
(n=37), \mathrm{cm}^{3} \\
(\mathrm{SD})\end{array}$ & $t$ & $p$ & Males $(n=31)$ & Females $(n=37)$ & $t$ & $p$ \\
\hline ICV & $625.7(60.7)$ & $608.2(55.2)$ & & - & & & & \\
\hline TBV & 450.8 (43.6) & $442.6(40.3)$ & & - & & & & \\
\hline TWM & $211.1(18.7)$ & $205.3(18.0)$ & & - & 0.47 & 0.46 & & - \\
\hline TGM & $240.6(26.2)$ & $237.4(23.8)$ & & - & 0.60 & 0.61 & -2.26 & $0.027 *$ \\
\hline Left & $122.3(14.9)$ & $116.6(9.2)$ & & - & 0.27 & 0.27 & & - \\
\hline Right & $122.7(15.0)$ & $116.4(9.0)$ & 2.067 & $0.044 *$ & 0.27 & 0.27 & & - \\
\hline $\mathrm{TCV}$ & $222.0(25.0)$ & $219.8(22.6)$ & & - & 0.49 & 0.50 & & - \\
\hline Frontal lobe & 80.8 (10.9) & $76.1(6.8)$ & 2.107 & $0.040 *$ & 0.33 & 0.33 & & - \\
\hline Left & $40.3(5.6)$ & $37.9(3.3)$ & & - & 0.17 & 0.16 & & - \\
\hline Right & $40.5(5.3)$ & $38.2(3.5)$ & 2.094 & $0.041^{*}$ & 0.17 & 0.16 & & - \\
\hline Parietal lobe & $43.4(5.5)$ & $41.3(3.7)$ & & - & 0.18 & 0.18 & -2.373 & $0.021 *$ \\
\hline Left & $21.9(2.8)$ & $20.9(2.0)$ & & - & 0.09 & 0.09 & -2.443 & $0.017 *$ \\
\hline Right & $21.6(2.8)$ & $20.4(1.9)$ & & - & 0.09 & 0.09 & & - \\
\hline Temporal lobe & $47.9(5.8)$ & $45.4(3.9)$ & 2.098 & $0.041^{*}$ & 0.19 & 0.20 & & - \\
\hline Left & $22.0(2.7)$ & $20.9(1.8)$ & 2.02 & $0.049 *$ & 0.09 & 0.09 & & - \\
\hline Right & $25.9(3.2)$ & $24.5(2.2)$ & 2.09 & $0.042 *$ & 0.11 & 0.11 & -2.367 & $0.021 *$ \\
\hline Occipital lobe & 54.8 (7.6) & $52.7(4.8)$ & & - & 0.23 & 0.22 & & - \\
\hline Left & $34.3(4.7)$ & $33.3(3.2)$ & & - & 0.14 & 0.14 & & - \\
\hline Right & $25.7(3.6)$ & $24.5(2.2)$ & & - & 0.11 & 0.11 & & - \\
\hline
\end{tabular}

Relative total volumes of grey and white matter were derived from relating absolute volumes to TBV and lobar absolute volumes to TGM. Independent samples $t$ test was used with normally distributed values and Mann-Whitney $U$ test with non-normally distributed values. Only statistically significant $(p<0.05)$ results are listed and marked with an asterisk

$I C V$ intracranial volume, $T B V$ total brain volume, $T W M$ total white matter, $T G M$ total gray matter volume, $T C V$ total cortical volume, - no association/ non-significant

development of any communication skills. However, discrepant evidence exists also on hemispheric differences that contradict with the rightward asymmetry findings. Gilmore at al. found left-larger-than-right hemispheric asymmetry in infants and also a laterality difference that was greater in females: the left occipital and prefrontal regions being larger than the right, although the result for the prefrontal area was non-significant and they reported findings on absolute volumes only (Gilmore et al. 2007). While our results do not concur with these hemispheric asymmetry findings, we did see a coinciding pattern of the left occipital lobe being larger than the right.

In this study population, age was not associated with asymmetry. Nevertheless, different hemispheric growth patterns might appear later, as it has been seen in longitudinal settings in older children (Matsuzawa et al. 2001; Tanaka et al. 2013). This may be a consequence of the different hierarchical functions of the brain networks: later maturing parts contain association areas that integrate input from different sensory sources (Tanaka et al. 2013). In conclusion, the detected similar asymmetries in parietal, temporal and occipital lobes in both sexes are highly accordant with previous findings and confirm the already existing observations on early asymmetry. These brain alterations possibly support overall development, yet more longitudinal research is needed on the matter.

\section{Strengths and limitations}

A key strength of the present study was that the individuals were scanned soon after birth to prevent the confounding effects of postnatal factors. The age variance was nevertheless broad enough to justify the age-volume correlation analyses. In addition, only full-term infants were included minimizing the complex and possibly confounding effects of prematurity and other pregnancy complications. The same scanner and scanning protocol were used for all individuals. The generalizability of these results is subject to certain limitations. For instance, lobar volumes are a gross, yet robust, parameter to evaluate brain growth let alone brain development, as volumetric differences do not reveal directly the functional development of the nervous system. Additionally, the data included only full-term Finnish infants and may not be generalizable to other infant groups or nationalities. As the study is 
A

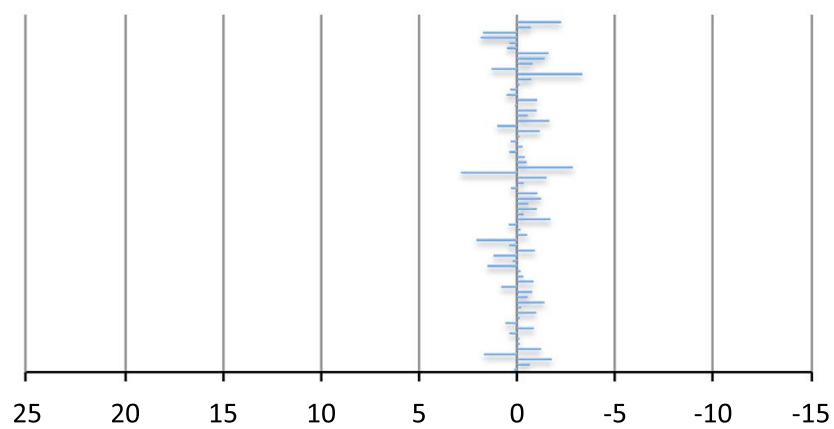

C

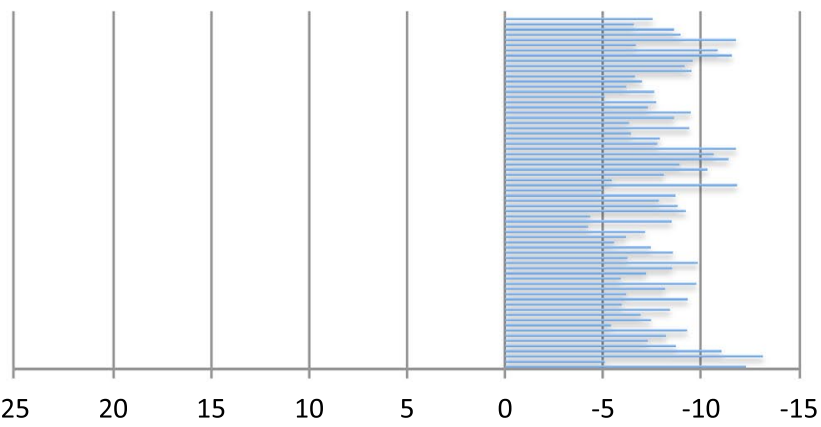

B

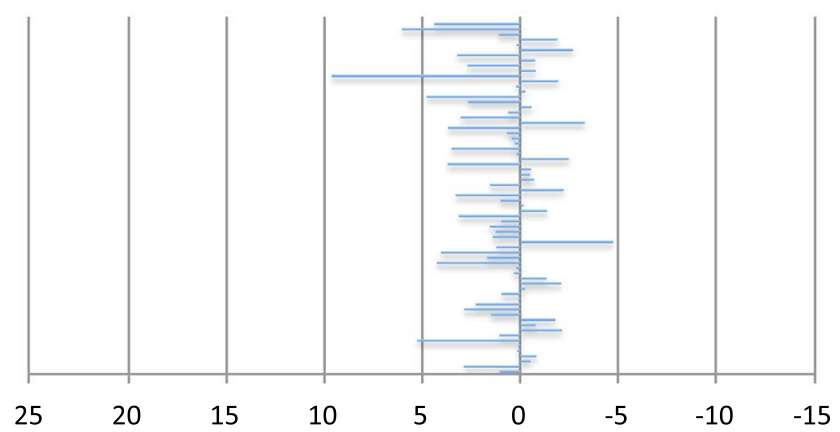

D

Occipital Asymmetry

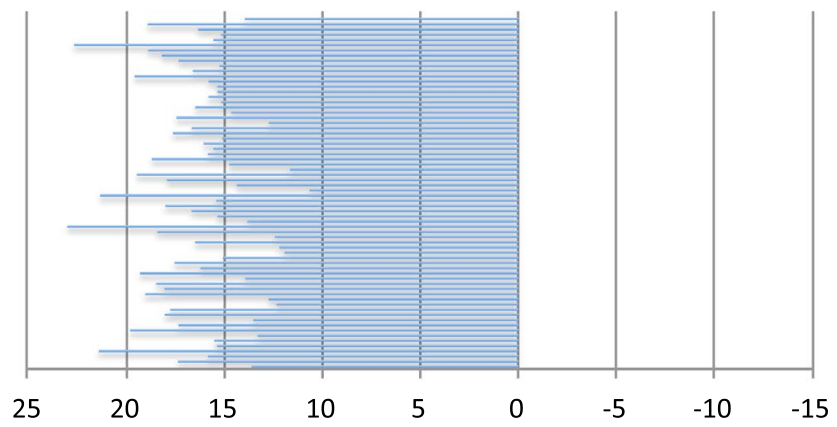

Asymmetry Index: Positive values indicate the left hemisphere and negative values the right hemisphere

Fig. 5 Lobar asymmetry retrieved from subtraction of the right side from the left and relating the result to the whole volume of the lobe. The negative values represent the right hemisphere and the positive values the left hemisphere. a Frontal lobe (result non-significant); b parietal lobe $(p<0.003)$; c temporal lobe $(p<0.0001)$; d occipital lobe $(p<0.0001)$

Table 6 Cerebral asymmetry

\begin{tabular}{lccrrll}
\hline Brain volume & Left mean (SD) & Right mean (SD) & AI, L $>$ R \% & $t$ & $p$ & $p$ (REL) \\
\hline TGM $\left(\mathrm{cm}^{3}\right)$ & $119.2(12.4)$ & $119.2(12.4)$ & -0.038 & $-0.43-$ & - \\
Frontal $\left(\mathrm{cm}^{3}\right)$ & $39.0(4.6)$ & $39.2(4.6)$ & -0.263 & $-1.93-$ & - \\
Parietal $\left(\mathrm{cm}^{3}\right)$ & $21.3(2.4)$ & $21.0(2.4)$ & 0.899 & 3.05 & $\mathbf{0 . 0 0 3}^{*}$ & $\mathbf{0 . 0 0 3}^{*}$ \\
Temporal $\left(\mathrm{cm}^{3}\right)$ & $21.4(2.3)$ & $25.2(2.8)$ & -8.131 & -32.93 & $<\mathbf{0 . 0 0 0 1}^{*}$ & $<\mathbf{0 . 0 0 0 1}^{*}$ \\
Occipital $\left(\mathrm{cm}^{3}\right)$ & $33.8(4.0)$ & $25.0(2.9)$ & 16.282 & 51.91 & $<\mathbf{0 . 0 0 0 1}^{*}$ & $<\mathbf{0 . 0 0 0 1} *$ \\
\hline
\end{tabular}

Comparison between left and right hemisphere. $p$ values are derived from one sample $t$ test. Significance level $(p<0.05)$ is marked by an asterisk. Bonferroni correction for multiple comparisons corresponding to $p<0.01 . p$ values meeting the significance criteria are bolded

AI Asymmetry Index, $T B V$ total brain volume, $T G M$ total gray matter volume, $p(R E L)$ TGM controlled with TBV and lobar volumes with TGM; - non-significant

cross-sectional, the possible functional correlates remain to be studied in the future. Finally, the narrow age range inhibited further investigation of potential sex differences in brain growth patterns. 


\section{Conclusion}

In this study, we examined lobar asymmetry and the relations of brain volumes to infant age (2-5 weeks of age) and sex. We discovered that lobar asymmetry was present in the infant brain and similar in both sexes; however, the degree of asymmetry had no association with infant age. As expected, age predicted positively the volume of gray matter and negatively that of the white matter reflecting the different growth rates and patterns of both brain matters. We also found some regional gray matter volume differences between sexes suggesting that sexual dimorphism in brain development may be seen already at this stage, but only in some parts of the brain. This research extends previous information on early brain development.

Acknowledgements Open access funding provided by University of Turku (UTU) including Turku University Central Hospital. We thank our radiographer Krisse Kuvaja for performing the imaging and the FinnBrain staff as well as all the participating families.

Author contributions Author SJL received research grants from Maire Taponen Foundation, Juho Vainio Foundation and the Hospital District of Southwest Finland (State research grant). JJT was supported by the Hospital District of Southwest Finland (State research grant) (data collection and data analysis) and Emil Aaltonen Foundation (interpretation of the data and manuscript revision). HM was supported by Sgirid Jusélius Foundation. Author NMS was funded by the Signe and Ane Gyllenberg Foundation as well as the Hospital District of Southwest Finland (State research grant). LK was supported by NARSAD Brain and Behaviour Research Foundation YI Grant 1956 (design and data collection), Finnish Medical Foundation (design and data collection) and Yrjö Jahnsson Foundation (interpretation of the data and manuscript revision). Author HK was funded by Finnish Academy (nos. 264363, 253270, 134950) (design and conduct of the study; collection, management, analysis, and interpretation of the data) and Jane and Aatos Erkko Foundation (management, analysis, and interpretation of the data).

Funding This study was funded by Maire Taponen Foundation, Juho Vainio Foundation, the Hospital District of Southwest Finland (State research grant), Emil Aaltonen Foundation, Signe and Ane Gyllenberg Foundation, Sigrid Jusélius Foundation, NARSAD Brain and Behaviour Research Foundation YI Grant 1956, Finnish Medical Foundation, Yrjö Jahnsson Foundation, Finnish Academy (nos. 264363, 253270, 134950) and Jane and Aatos Erkko Foundation.

\section{Compliance with ethical standards}

Conflict of interest The authors declare that they have no conflict of interest.

Ethical approval All procedures performed in studies involving human participants were in accordance with the ethical standards of Ethics Committee of the Hospital District of Southwest Finland and with the 1964 Helsinki declaration and its later amendments or comparable ethical standards.

Informed consent Informed consent was obtained from all individual participants included in the study.
Open Access This article is distributed under the terms of the Creative Commons Attribution 4.0 International License (http://creativeco mmons.org/licenses/by/4.0/), which permits unrestricted use, distribution, and reproduction in any medium, provided you give appropriate credit to the original author(s) and the source, provide a link to the Creative Commons license, and indicate if changes were made.

\section{References}

Aanes S, Bjuland KJ, Skranes J, Løhaugen GCC (2015) Memory function and hippocampal volumes in preterm born very-lowbirth-weight (VLBW) young adults. NeuroImage 105:76-83. https://doi.org/10.1016/j.neuroimage.2014.10.023

Arthurs OJ, Edwards A, Austin T, Graves MJ, Lomas DJ (2012) The challenges of neonatal magnetic resonance imaging. Pediatr Radiol 42(10):1183-1194. https://doi.org/10.1007/s0024 7-012-2430-2

Bora S, Pritchard VE, Chen Z, Inder TE, Woodward LJ (2014) Neonatal cerebral morphometry and later risk of persistent inattention/hyperactivity in children born very preterm. J Child Psychol Psychiatry Allied Discip 55(7):828-838. https://doi. org/10.1111/jcpp.12200

Dai Y, Shi F, Wang L, Wu G, Shen D (2013) iBEAT: A toolbox for infant brain magnetic resonance image processing. Neuroinformatics 11(2):211-225. https://doi.org/10.1007/s1202 1-012-9164-Z

Dean DC, Planalp EM, Wooten W, Schmidt CK, Kecskemeti SR, Frye C, Davidson RJ (2018) Investigation of brain structure in the 1-month infant. Brain Struct Funct. https://doi.org/10.1007/ s00429-017-1600-2

Deoni S, Mercure E, Blasi A, Gasston D, Thomson A, Johnson M, Murphy D (2011) Mapping infant brain myelination with magnetic resonance imaging. J Neurosci 31(2):784-791. https://doi. org/10.1523/JNEUROSCI.2106-10.2011

Gilmore JH, Lin W, Prastawa MW, Looney CB, Sampath Y, Vetsa K, Gerig G (2007) Regional gray matter growth, sexual dimorphism, and cerebral asymmetry in the neonatal brain. J Neurosci 27(6):1255-1260. https://doi.org/10.1523/JNEUR OSCI.3339-06.2007

Guo JY, Isohanni M, Miettunen J, Jääskeläinen E, Kiviniemi V, Nikkinen J, Murray GK (2016) Brain structural changes in women and men during midlife. Neurosci Lett 615:107-112. https://doi. org/10.1016/j.neulet.2016.01.007

Haukvik UK, Rimol LM, Roddey JC, Hartberg CB, Lange EH, Vaskinn A, Agartz I (2014) Normal birth weight variation is related to cortical morphology across the psychosis spectrum. Schizophr Bull 40(2):410-419. https://doi.org/10.1093/schbu $1 /$ sbt005

Holland D, Chang L, Ernst TM, Curran M, Buchthal SD, Alicata D, Dale AM (2014) Structural growth trajectories and rates of change in the first 3 months of infant brain development. JAMA Neurol 71(10):1266-1274. https://doi.org/10.1001/jamaneurol.2014.1638

Irimia A, Van Horn JD (2013) The structural, connectomic and network covariance of the human brain. NeuroImage 66:489-499. https://doi.org/10.1016/j.neuroimage.2012.10.066

Knickmeyer RC, Gouttard S, Kang C, Evans D, Wilber K, Smith JK, Gilmore JH (2008a) A structural MRI study of human brain development from birth to 2 years. J Neurosci 28(47):1217612182. https://doi.org/10.1523/JNEUROSCI.3479-08.2008

Knickmeyer R, Gouttard S, Kang C, Evans D, Wilber K, Smith JK, Gilmore J (2008b) A structural MRI study of human brain development from birth to 2 years. J Neurosci 28(47):1217612182. https://doi.org/10.1523/JNEUROSCI.3479-08.2008 
Knickmeyer RC, Xia K, Lu Z, Ahn M, Jha SC, Zou F, Gilmore JH (2017) Impact of demographic and obstetric factors on infant brain volumes: a population neuroscience study. Cereb Cortex 5(Suppl 1):187. https://doi.org/10.1093/cercor/bhw331

Koolschijn PCMP, Crone EA (2013) Sex differences and structural brain maturation from childhood to early adulthood. Dev Cogn Neurosci 5:106-118. https://doi.org/10.1016/j.den.2013.02.003

Lerch JP, Worsley K, Shaw WP, Greenstein DK, Lenroot RK, Giedd J, Evans AC (2006) Mapping anatomical correlations across cerebral cortex (MACACC) using cortical thickness from MRI. NeuroImage 31(3):993-1003. https://doi.org/10.1016/j.neuro image.2006.01.042

Mark LP, Daniels DL, Naidich TP, Hendrix LE (1995) Limbic connections. Am J Neuroradiol 16(6):1303-1306

Matsuzawa J, Matsui M, Konishi T, Noguchi K, Gur RC, Bilker W, Miyawaki T (2001) Age-related volumetric changes of brain gray and white matter in healthy infants and children. Cereb Cortex 11(4):335-342

Mills KL, Tamnes CK (2014) Methods and considerations for longitudinal structural brain imaging analysis across development. Dev Cogn Neurosci 9:172-190. https://doi.org/10.1016/j. den.2014.04.004

Munakata S, Okada T, Okahashi A, Yoshikawa K, Usukura Y, Makimoto M, Okuhata Y (2013) Gray matter volumetric MRI differences late-preterm and term infants. Brain Dev 35(1):10-16. https ://doi.org/10.1016/j.braindev.2011.12.011

Østgård HF, Løhaugen GCC, Bjuland KJ, Rimol LM, Brubakk A-M, Martinussen M, Skranes J (2014) Brain morphometry and cognition in young adults born small for gestational age at term. J Pediatr 165(5):921-927.e1. https://doi.org/10.1016/j.jpeds .2014.07.045

Peterson BS, Vohr B, Staib LH, Cannistraci CJ, Dolberg A, Schneider KC, JL, R (2000) Regional brain volume abnormalities and longterm cognitive outcome in preterm infants. JAMA 284(15):1939. https://doi.org/10.1001/jama.284.15.1939

Rajagopalan V, Scott J, Habas PA, Kim K, Rousseau F, Glenn OA, Studholme C (2011) Mapping directionality specific volume changes using tensor based morphometry: an application to the study of gyrogenesis and lateralization of the human fetal brain. NeuroImage 63(2):947-958. https://doi.org/10.1016/j.neuroimage .2012 .03 .092
Rooks VJ, Eaton JP, Ruess L, Petermann GW, Keck-Wherley J, Pedersen RC (2008) Prevalence and evolution of intracranial hemorrhage in asymptomatic term infants. AJNR Am J Neuroradiol 29(6):1082-1089. https://doi.org/10.3174/ajnr.A1004

Stiles J, Jernigan TL (2010) The basics of brain development. Neuropsychol Rev 20(4):327-348. https://doi.org/10.1007/s1106 5-010-9148-4

Tanaka C, Matsui M, Uematsu A, Noguchi K, Miyawaki T (2013) Developmental trajectories of the fronto-temporal lobes from infancy to early adulthood in healthy individuals. Dev Neurosci, 34(6). https://doi.org/10.1159/000345152

Thompson DK, Warfield SK, Carlin JB, Pavlovic M, Wang HX, Bear M, Inder TE (2007) Perinatal risk factors altering regional brain structure in the preterm infant. Brain 130(3):667-677

Uematsu A, Matsui M, Tanaka C, Takahashi T, Noguchi K, Suzuki M, Nishijo H (2012) Developmental trajectories of amygdala and hippocampus from infancy to early adulthood in healthy individuals. PloS One 7(10):e46970. https://doi.org/10.1371/journ al.pone.0046970

van Soelen ILC, Brouwer RM, Peper JS, van Beijsterveldt TCEM, van Leeuwen M, de Vries LS, Boomsma DI (2010) Effects of Gestational age and birth weight on brain volumes in healthy 9 year-old children. J Pediatr 156(6):896-901. https://doi.org/10.1016/j.jpeds .2009 .12 .052

Walhovd KB, Fjell AM, Brown TT, Kuperman JM, Chung Y, Hagler DJ, Pediatric Imaging, Neurocognition, and Genetic Study G (2012) ... Long-term influence of normal variation in neonatal characteristics on human brain development. Proc Natl Acad Sci USA 109(49):20089-20094. https://doi.org/10.1073/pnas.12081 80109

Walhovd KB, Fjell AM, Giedd J, Dale AM, Brown TT (2016) Through thick and thin: a need to reconcile contradictory results on trajectories in human cortical development. Cereb Cortex 27(2):bhv301. https://doi.org/10.1093/cercor/bhv301

Whitby E, Griffiths P, Rutter S, Smith M, Sprigg A, Ohadike P, Paley M (2004) Frequency and natural history of subdural haemorrhages in babies and relation to obstetric factors. Lancet 363(9412):846851. https://doi.org/10.1016/S0140-6736(04)15730-9 